\title{
When all points are generic for ergodic measures
}

by

\author{
Tomasz DOWNAROWICZ and Benjamin WEISS \\ Presented by Feliks PRZYTYCKI
}

\begin{abstract}
Summary. We establish connections between several properties of topological dynamical systems, such as:

- every point is generic for an ergodic measure,

- the map sending points to the measures they generate is continuous,

- the system splits into uniquely (alternatively, strictly) ergodic subsystems,

- the map sending ergodic measures to their topological supports is continuous,

- the Cesàro means of every continuous function converge uniformly.
\end{abstract}

1. Introduction. It follows from the pointwise ergodic theorem that for a topological dynamical system $(X, T)$ with an ergodic invariant measure $\mu$, a.e. point $x \in X$ is generic for $\mu$, i.e. for any continuous function $f: X \rightarrow \mathbb{R}$, the Cesàro means

$$
\frac{1}{n} \sum_{i=0}^{n-1} f\left(T^{i} x\right)
$$

converge to $\int f d \mu$. In 1952, Oxtoby [O] showed that in a uniquely ergodic topological dynamical system $(X, T)$ every point $x \in X$ is generic for the unique invariant measure $\mu$ carried by $X$, and moreover the above convergence is uniform. This implies, in particular, that any topological system $(X, T)$ which splits as a disjoint union of uniquely ergodic subsystems has the property that every point $x \in X$ is generic for an ergodic measure (we will say that $(X, T)$ is pointwise ergodic). The converse need not hold: in 1981,

2020 Mathematics Subject Classification: Primary 37B05, 37B20; Secondary 37A25.

Key words and phrases: topological dynamical system, measure-preserving system, ergodic measure, semicontinuous partition, uniform system, strictly uniform system, semisimple system, generic point.

Received 13 January 2021.

Published online 25 January 2021. 
Y. Katznelson and B. Weiss [KW] provided an example of a pointwise ergodic system with uncountably many ergodic measures, but just one minimal subset (this makes splitting into uniquely ergodic subsystems impossible).

In 1970, W. Krieger, following R. Jewett, established that every ergodic measure-preserving system has a strictly ergodic (i.e. uniquely ergodic and minimal) topological model. It is worth noticing that once a uniquely ergodic model is found, a strictly ergodic model is easily obtained by taking the (unique) minimal subsystem of that model. Four years later, G. Hansel generalized the Jewett-Krieger Theorem to nonergodic systems as follows: every measure-preserving system has a topological model $(X, T)$ which splits as a union of strictly ergodic subsystems, and moreover the Cesàro means of any continuous function converge uniformly on $X$ (he called the latter property uniformity). It has to be pointed out that in the nonergodic case, finding a model which splits into uniquely ergodic subsystems is by far insufficient: the minimal subsystems of the uniquely ergodic components need not unite to a closed set, and the uniformity, which holds individually on each uniquely ergodic component, need not coordinate to a global uniformity on $X$. Thus, a model which splits into uniquely ergodic subsystems neither automatically provides a model which splits into strictly ergodic subsystems, nor one which is uniform. This is why Hansel's result can be considered much stronger than just a straightforward generalization of the Jewett-Krieger Theorem.

We are interested in studying those properties of non-uniquely-ergodic topological dynamical systems which in the uniquely (alternatively, strictly) ergodic case are automatic:

- pointwise ergodicity,

- splitting into uniquely ergodic subsystems,

- splitting into strictly ergodic subsystems,

- uniformity.

Two more properties emerge naturally in the context of pointwise ergodicity (both hold trivially in uniquely ergodic systems):

- continuity of the mapping that associates to each point $x \in X$ the ergodic measure for which $x$ is generic, and

- continuity of the set-valued function that associates to each ergodic measure its topological support.

In this paper we establish a diagram of implications (and equivalences) between these properties (and some of their conjunctions). Our culminating result is a characterization of uniformity in terms of the topological organization of the uniquely ergodic components. 
2. Some terminological conventions. Throughout this paper, by a topological dynamical system we will mean a pair $(X, T)$, where $X$ is a compact metric space and $T: X \rightarrow X$ is a homeomorphism. We will denote by $\mathcal{M}(X)$ and $\mathcal{M}_{T}(X)$ the collections of all Borel probability measures on $X$ and of all invariant $\left({ }^{1}\right)$ Borel probability measures on $X$, respectively. By saying "measure" we will always mean a Borel probability, since no other measures will be considered.

It is well known that $\mathcal{M}_{T}(X)$ endowed with the weak-star topology is a nonempty metrizable simplex $\left[\left(^{2}\right)\right.$ and that the extreme points of $\mathcal{M}_{T}(X)$ are the ergodic measures $\left(^{3}\right)$. The set of ergodic measures will be denoted by $\mathcal{M}_{T}^{\mathrm{e}}(X)$. When $\mathcal{M}_{T}(X)$ is a singleton (equivalently, when $\mathcal{M}_{T}(X)=$ $\left.\mathcal{M}_{T}^{\mathrm{e}}(X)\right)$, the system $(X, T)$ is called uniquely ergodic. A system $(X, T)$ is called strictly ergodic if it is uniquely ergodic and minimal $\left({ }^{4}\right)$. It is elementary to see that if $(X, T)$ is uniquely ergodic then $X$ contains a unique minimal subset $M$ which equals the topological support $\left(^{5}\right)$ of the unique invariant measure. Then $(M, T)\left(^{6}\right)$ is a strictly ergodic system and $\mathcal{M}_{T}(X)=\mathcal{M}_{T}(M)$.

By a measure-preserving system we will understand a quadruple $(X, \Sigma, \mu, T)$, where $(X, \Sigma, \mu)$ is a standard (Lebesgue) probability space and $T: X \rightarrow X$ is a measure automorphism. When a topological dynamical system $(X, T)$ is considered with a fixed invariant measure $\mu \in \mathcal{M}_{T}(X)$, it becomes a measure-preserving system $(X, \mu, T)$. The indication of the sigma-algebra is omitted intentionally, as this role will always be played by the sigma-algebra of Borel sets of $X$ (technically, in order to have a standard probability space, we need to consider the Borel sigma-algebra completed with respect to $\mu$ ).

Two measure-preserving systems, say $(X, \Sigma, \mu, T)$ and $(\bar{X}, \bar{\Sigma}, \bar{\mu}, \bar{T})$, are said to be isomorphic if there exists an isomorphism $\phi: X \rightarrow \bar{X}$ of the measure spaces $(X, \Sigma, \mu)$ and $(\bar{X}, \bar{\Sigma}, \bar{\mu})$ which intertwines the actions of $T$ and $\bar{T}$, i.e. $\phi \circ T=\bar{T} \circ \phi$. In that case, by a commonly accepted abuse of terminology, we will briefly say that $\mu$ and $\bar{\mu}$ are isomorphic.

$\left({ }^{1}\right)$ In a topological dynamical system $(X, T)$, a measure $\mu$ on $X$ is invariant if $\int f d \mu=$ $\int f \circ T d \mu$ for every continuous $f: X \rightarrow \mathbb{R}$.

$\left({ }^{2}\right)$ A metrizable simplex is a compact convex metric space such that every point is the barycenter of a unique probability measure supported by the set of extreme points.

$\left({ }^{3}\right)$ A measure $\mu \in \mathcal{M}_{T}(X)$ is ergodic if all $T$-invariant Borel sets have measure zero or one.

$\left(^{4}\right)$ A topological dynamical system is minimal if it has no proper closed invariant subsets.

$\left({ }^{5}\right)$ The topological support of a measure is the smallest closed set of measure 1 .

$\left({ }^{6}\right)$ We write $T$, but in fact, we mean here the restriction of $T$ to $M$. 
Two topological dynamical systems $(X, T)$ and $(\bar{X}, \bar{T})$ are topologically conjugate (briefly conjugate) if there exists a homeomorphism $\phi: X \rightarrow \bar{X}$ which intertwines the actions of $T$ and $\bar{T}$.

A topological dynamical system $(\bar{X}, \bar{T})$ is called a model for a measurepreserving system $(X, \Sigma, \mu, T)$ if there exists an invariant measure $\bar{\mu} \in$ $\mathcal{M}_{\bar{T}}(\bar{X})$ isomorphic to $\mu$. If $(\bar{X}, \bar{T})$ is also uniquely ergodic, we call it a uniquely ergodic model of $(X, \Sigma, \mu, T)$. If $(X, T)$ is strictly ergodic, we call it a strictly ergodic (or Jewett-Krieger) model of $(X, \Sigma, \mu, T)$. If $(\bar{X}, \bar{T})$ is a uniquely ergodic model of $(X, \Sigma, \mu, T)$, then $(\bar{M}, \bar{T})$ is a strictly ergodic model of $(X, \Sigma, \mu, T)$, where $\bar{M}$ is the unique minimal subset of $\bar{X}$.

\section{Upper and lower semicontinuous partitions and multifunc-} tions. Let $X$ be a compact metric space. We denote by $2^{X}$ the space of all compact subsets of $X$ topologized by the Hausdorff metric (then $2^{X}$ is also a compact metric space).

Definition 3.1. A partition $\mathcal{P}$ of $X$ with closed atoms is upper (resp. lower) semicontinuous if, whenever $\left(A_{n}\right)_{n \geq 1}$ is a sequence of atoms of $\mathcal{P}$, converging (in the Hausdorff metric) to a set $S$, then $S$ is contained in (resp. contains) an atom of $\mathcal{P}$. If $\mathcal{P}$ is both upper and lower semicontinuous, it is called continuous.

The following facts are true. The last statement, for which we give no reference, is an easy exercise.

\section{Proposition 3.2.}

(1) [HY, Theorem 3-31] Let $f: X \rightarrow Y$ be any function between any spaces. Define the fiber partition as the partition of $X$ whose atoms are the sets $f^{-1}(y), y \in Y$. If $X$ and $Y$ are compact metric and $f$ is continuous then the fiber partition has closed atoms and is upper semicontinuous.

(2) [HY, Theorem 3-34] Any partition $\mathcal{P}$ of a compact metric space X, with closed atoms and upper semicontinuous, equals the fiber partition of some continuous function $f: X \rightarrow Y$ onto a compact metric space $Y$.

(3) The common refinement of any collection of upper semicontinuous partitions with closed atoms has closed atoms and is upper semicontinuous.

Definition 3.3. A set-valued function $f: Y \rightarrow 2^{X}$, where $X$ and $Y$ are compact metric spaces, is upper (resp. lower) semicontinuous if, whenever $\left(y_{n}\right)_{n \geq 1}$ is a sequence in $Y$ converging to some $y \in Y$ and $f\left(y_{n}\right) \rightarrow S \in 2^{X}$, then $S$ is contained in (resp. contains) $f(y)$. 
It is elementary to see that a function $f: Y \rightarrow 2^{X}$ is continuous if and only if it is both upper and lower semicontinuous.

Note the following obvious fact:

Proposition 3.4. If $f: Y \rightarrow 2^{X}$, where $X$ and $Y$ are compact metric spaces, is upper (resp. lower) semicontinuous, and the collection of values, $\{f(y): y \in Y\}$, happens to be a partition of $X$, then this partition is upper (resp. lower) semicontinuous.

We remark that the converse need not hold. Indeed, suppose that a setvalued function $f: Y \rightarrow 2^{X}$ is such that $\{f(y): y \in Y\}$ is an upper (resp. lower) semicontinuous partition of $X$. For any bijection $g: X \rightarrow X$, the partition by images of the composition $f \circ g$ is the same as that for $f$, while the composition may destroy any continuity properties of the function.

A very useful class of lower semicontinuous set-valued functions is provided by the following fact, whose elementary proof we skip:

Proposition 3.5. Let $X$ be a compact metric space and let $\mathcal{M}(X)$ denote the set of all probability measures on $X$, equipped with the weak-star topology. Then the function supp : $\mathcal{M}(X) \rightarrow 2^{X}$, assigning to each measure its topological support, is lower semicontinuous.

4. Strict uniformity and related notions. Our goal is to introduce and compare several properties, of similar flavor, of topological dynamical systems. Although none of these properties is new and they appear in various contexts in the literature, to our knowledge they have never been given short names (exception: "semisimple"). For convenience of our further discussion, we introduce such names below.

Definition 4.1. A topological dynamical system $(X, T)$ will be called:

(I) Pointwise ergodic if every point $x \in X$ is generic for an ergodic measure. In this case, we let $\Phi: X \rightarrow \mathcal{M}_{T}^{\mathrm{e}}(X)$ denote the map associating to each point $x \in X$ the ergodic measure for which $x$ is generic. Also, we let $\mathcal{P}$ denote the fiber partition of the function $\Phi$.

(II) Semisimple if $X$ is a union of minimal sets.

(III) Partitionable if $X$ is a disjoint union of closed invariant uniquely ergodic sets (we will say uniquely ergodic subsystems).

(IV) Strictly partitionable if $X$ is a union of strictly ergodic subsystems.

(V) Continuously pointwise ergodic if it is pointwise ergodic and $\Phi$ is continuous.

(VI) Continuously strictly pointwise ergodic if it is continuously pointwise ergodic and semisimple. 
We begin the analysis of the connections between the notions (I)-(VI) by some fairly obvious observations:

PROPOSITION 4.2.

(1) A system $(X, T)$ is partitionable if and only if it is pointwise ergodic and $\mathcal{P}$ has closed atoms.

(2) A system $(X, T)$ is strictly partitionable if and only if it is pointwise ergodic and semisimple.

(3) A system $(X, T)$ is continuously pointwise ergodic if and only if it is partitionable and $\mathcal{P}$ is upper semicontinuous.

(4) A system $(X, T)$ is continuously strictly pointwise ergodic if and only if it is strictly partitionable and $\mathcal{P}$ is upper semicontinuous.

(5) If $(X, T)$ is continuously pointwise ergodic then $\mathcal{M}_{T}^{\mathrm{e}}(X)$ is compact.

Proof. (1) A partitionable system splits into uniquely ergodic subsystems and each of these subsystems is pointwise ergodic (see [0]). So the whole system is pointwise ergodic as well. The atoms of $\mathcal{P}$ coincide with the uniquely ergodic subsystems, so they are closed. The converse implication is obvious: the atoms of $\mathcal{P}$, if closed, are uniquely ergodic subsystems.

(2) A strictly partitionable system is pointwise ergodic by (1), and semisimple by definition. If a semisimple system is pointwise ergodic then so is each of its minimal subsystems. A pointwise ergodic minimal system is uniquely ergodic (see [KW, Proposition 3.2]), which implies strict partitionability.

(3) The fact that continuity of $\Phi$ implies closed atoms and upper semicontinuity of $\mathcal{P}$ follows directly from Proposition 3.2(1). Suppose that $\mathcal{P}$ has closed atoms and is upper semicontinuous, and consider a sequence $\left(x_{n}\right)_{n \geq 1}$ of points in $X$ converging to a point $x \in X$. Denote $\mu_{n}=\Phi\left(x_{n}\right), \mu=\Phi(x)$, and let $A_{n}$ and $A$ denote the atoms of $\mathcal{P}$ containing $x_{n}$ and $x$, respectively. By passing to a subsequence, we may assume that the $\mu_{n}$ converge to some invariant measure $\mu^{\prime} \in \mathcal{M}_{T}(X)$ and that the $A_{n}$ converge in $2^{X}$ to some compact set $A^{\prime} \subset X$. By upper semicontinuity of $\mathcal{P}$, we have $A^{\prime} \subset A$. On the other hand, by lower semicontinuity of the set-valued function supp on $\mathcal{M}_{T}(X)$, we also have $\operatorname{supp}\left(\mu^{\prime}\right) \subset A^{\prime}$, which implies that $\mu^{\prime}$ is carried by the atom $A$. Since $A$ carries only one invariant measure $\mu=\Phi(x)$, we find that $\mu^{\prime}=\mu$, which concludes the proof of continuity of $\Phi$.

(4) is (3) applied to semisimple systems.

(5) is obvious: a continuous image of a compact space is compact.

In addition to the notation (I)-(VI), let us denote by $(\mathrm{P})$ the condition that $\mathcal{P}$ has closed atoms and is upper semicontinuous (equivalently, that $\Phi$ is continuous), by (B) the property that $\mathcal{M}_{T}^{\mathrm{e}}(X)$ is compact (in other words, 
that $\mathcal{M}_{T}(X)$ is a Bauer simplex $\left({ }^{7}\right)$, and by $(\mathrm{S})$ the condition that the set-valued function supp is continuous on $\mathcal{M}_{T}^{\mathrm{e}}(X)$.

The property $(\mathrm{P})$ has a special interpretation, which follows immediately from Proposition $3.2(2)$ :

Proposition 4.3. Let $(X, T)$ be a pointwise ergodic dynamical system. The partition $\mathcal{P}$ has closed atoms and is upper semicontinuous if and only if there exists a topological factor $\pi: X \rightarrow Y$ from $(X, T)$ to a system $(Y, S)$ such that $S$ is the identity on $Y$, and each fiber $\pi^{-1}(y)$ is uniquely ergodic. In this case, for each invariant measure $\mu \in \mathcal{M}_{T}(X), \pi$ coincides (up to measure) with the measure-theoretic factor map from $(X, \mu, T)$ onto the sigma-algebra of invariant sets.

We continue by establishing more equivalences and implications.

Proposition $4.4((\mathrm{VI}) \Leftrightarrow(\mathrm{IV}) \wedge(\mathrm{B}) \wedge(\mathrm{S}))$. A system $(X, T)$ is continuously strictly pointwise ergodic if and only if it is strictly partitionable, $\mathcal{M}_{T}^{\mathrm{e}}(X)$ is compact, and the set-valued function supp : $\mathcal{M}_{T}^{\mathrm{e}}(X) \rightarrow 2^{X}$ is continuous. The partition $\mathcal{P}$ is then continuous.

Proof. Assume that $(X, T)$ is continuously strictly pointwise ergodic. We already know that $\mathcal{M}_{T}^{\mathrm{e}}(X)$ is then compact. Let $\left(\mu_{n}\right)_{n \geq 1}$ be a sequence of ergodic measures converging to an ergodic measure $\mu$. Let $A_{n}$ and $A$ denote the topological supports of $\mu_{n}$ and $\mu$, respectively. Consider a sequence of points $x_{n} \in A_{n}$ converging to a point $x \in X$. Since $\Phi$ is continuous, the measures $\mu_{n}=\Phi\left(x_{n}\right)$ converge to $\mu=\Phi(x)$, i.e. $x$ is generic for $\mu$. Since $(X, T)$ is strictly partitionable, this implies that $x \in \operatorname{supp}(\mu)=A$. We have shown that supp is upper semicontinuous on $\mathcal{M}_{T}^{\mathrm{e}}(X)$. Since supp is lower semicontinuous on $\mathcal{M}(X)$, we have proved continuity of supp on $\mathcal{M}_{T}^{\mathrm{e}}(X)$.

Conversely, assume that $(X, T)$ is strictly partitionable, $\mathcal{M}_{T}^{\mathrm{e}}(X)$ is compact, and supp is continuous on $\mathcal{M}_{T}^{\mathrm{e}}(X)$. Then $\mathcal{P}$ equals the partition by images of the continuous set-valued function supp defined on a compact space, so it is continuous, in particular upper semicontinuous. Proposition $4.2(4)$ now implies that $(X, T)$ is continuously strictly pointwise ergodic.

We are interested in two other properties of topological dynamical systems, which have seemingly a much more "ergodic-theoretic" flavor than all the "topological" properties discussed so far. Nonetheless, we will be able to provide their topological charactarizations. Both notions have been introduced in $[\mathrm{H}]$, but the respective properties seem to originate in a much earlier work $\mathrm{O}$.

$\left({ }^{7}\right)$ A Bauer simplex is a simplex whose set of extreme points is closed. 


\section{DEFINITION 4.5.}

- A topological dynamical system $(X, T)$ is called uniform if, for every continuous function $f: X \rightarrow \mathbb{R}$, the Cesàro means

$$
A_{n}^{f}(x)=\frac{1}{n} \sum_{i=0}^{n-1} f\left(T^{i} x\right)
$$

converge uniformly on $X$.

- A system $(X, T)$ which is both uniform and semisimple is called strictly uniform.

Clearly, in a uniform system, the limit function $\tilde{f}=\lim _{n} A_{n}^{f}$ is continuous. As we have already mentioned several times, uniquely ergodic systems are uniform. We have the following nearly obvious implication:

Proposition 4.6. Any uniform system is continuously pointwise ergodic. Any strictly uniform system is continuously strictly pointwise ergodic.

Proof. Clearly, a uniform system $(X, T)$ is pointwise ergodic. It is now crucial to observe that the partition $\mathcal{P}$ equals the common refinement over all continuous functions $f: X \rightarrow \mathbb{R}$ of the fiber partitions of the limit functions $\tilde{f}$. Now, by Proposition $3.2(1,3), \mathcal{P}$ is upper semicontinuous, and by Proposition $4.2(3),(X, T)$ is continuously pointwise ergodic. The statement about strictly uniform systems follows from the above applied to semisimple systems.

The second part of Proposition 4.6 places strict uniformity at the top of the hierarchy of properties discussed in this paper, as the most restrictive one. A notable role of strict uniformity in the theory of dynamical systems is sanctioned by two theorems, the first of which is the celebrated JewettKrieger Theorem $([\mathrm{J}]$ and $[\mathrm{K}]$ ); the second, although vastly generalizes the first one, is much less commonly known.

Theorem 4.7 (Jewett-Krieger $\left({ }^{8}\right)$, 1970). Every ergodic measure-preserving system $(X, \Sigma, \mu, T)$ has a strictly ergodic topological model.

As a corollary, we find that

- every ergodic measure-preserving system has a strictly uniform topological model.

The second theorem is due to $\mathrm{G}$. Hansel $[\mathrm{H}]$, and generalizes the above to nonergodic measure-preserving systems:

$\left({ }^{8}\right)$ Jewett showed this assuming weak mixing, and Krieger relaxed this assumption to ergodicity. 
ThEOREM 4.8 (Hansel, 1974). Every measure-preserving system $(X, \Sigma, \mu, T)$ has a strictly uniform topological model.

Hansel's theorem has the following remarkable interpretation: the class of strictly uniform systems, although the smallest (among the classes discussed in this paper), is still rich enough to capture "the entire ergodic theory".

We will denote uniformity by $(\mathrm{U})$ and strict uniformity by ( $\mathrm{sU}$ ). The following theorem, which to our best knowledge has not been noted to date, constitutes the main result of this paper. Combined with the equivalence $(\mathrm{V}) \Leftrightarrow(\mathrm{IV}) \wedge(\mathrm{P})$, it characterizes the "ergodic" property of uniformity by means of "topological organization" of the uniquely ergodic subsystems.

Theorem $4.9((\mathrm{U}) \Leftrightarrow(\mathrm{V}),(\mathrm{sU}) \Leftrightarrow(\mathrm{VI}))$. A system $(X, T)$ is uniform if and only if it is continuously pointwise ergodic. It is strictly uniform if and only if it is continuously strictly pointwise ergodic.

Proof. In view of Proposition 4.6, we only need to prove that continuously pointwise ergodic systems are uniform. So, assume that $(X, T)$ is continuously pointwise ergodic. Fix any continuous function $f: X \rightarrow[0,1]$ and a point $x_{0} \in X$. Let $\mu_{0}=\Phi\left(x_{0}\right)$. Given $\varepsilon>0$, there exists an $n_{0}=n\left(x_{0}\right)$ such that

$$
\left|A_{n_{0}}^{f}\left(x_{0}\right)-\int f d \mu_{0}\right|<\varepsilon / 2 .
$$

Since $\left|A_{n_{0}}^{f}(x)-\int f d \mu\right|$ is a jointly continuous function of $(x, \mu)$, so the inequality $\left|A_{n_{0}}^{f}(x)-\int f d \mu\right|<\varepsilon / 2$ holds for all measures $\mu$ in a sufficiently small neighborhood $V_{0}$ of $\mu_{0}$ and all points $x$ in a sufficiently small neighborhood $U_{0}$ of $x_{0}$. By continuity of $\Phi$, we can choose $U_{0}$ so small that $\Phi\left(U_{0}\right) \subset V_{0}$. Then we have

$$
\left|A_{n_{0}}^{f}(x)-\int f d \mu_{x}\right|<\varepsilon / 2
$$

for all $x \in U_{0}$, where $\mu_{x}=\Phi(x)$. By compactness, $X$ is covered by finitely many neighborhoods $U_{i}$ created analogously (with the same $\varepsilon$ ) for some points $x_{i} \in X(i=1, \ldots, l)$. This cover defines finitely many numbers $n_{i}=$ $n\left(x_{i}\right)$. Let $n$ be any integer larger than

$$
M=\frac{2 \max \left\{n_{1}, \ldots, n_{l}\right\}}{\varepsilon} .
$$

Pick any $x \in X$. We can divide the forward orbit of $x$ of length $n$ into "portions", as follows: $x$ belongs to some $U_{i_{0}}$, so we choose $n_{i_{0}}$ as the length of the first portion. Next, $T^{n_{i}} x$ belongs to some $U_{i_{1}}$, so we choose $n_{i_{1}}$ as the length of the second portion. Next, $T^{n_{i_{0}}+n_{i_{1}}} x$ belongs to some $U_{i_{2}}$, so we choose $n_{i_{2}}$ as the length of the third portion. And so on, until we are left with a "tail" of some length $m<\max \left\{n_{1}, \ldots, n_{l}\right\}$, starting at $T^{n_{i_{0}}+n_{i_{1}}+\cdots+n_{i_{q}}} x$ 
for some $q \in \mathbb{N}$. We have

$$
\begin{aligned}
A_{n}^{f}(x)= & \frac{n_{i_{0}}}{n} A_{n_{i_{0}}} x+\sum_{j=1}^{q} \frac{n_{i_{j}}}{n} A_{n_{i_{j}}}^{f}\left(T^{n_{i_{0}}+n_{i_{1}}+\cdots+n_{i_{j-1}}} x\right) \\
& +\frac{m}{n} A_{m}^{f}\left(T^{n_{i_{1}}+n_{i_{2}}+\cdots+n_{i_{q}}} x\right) .
\end{aligned}
$$

Because all the points $T^{n_{i_{j}}} x$ are generic for the same measure $\mu_{x}$, we have

$$
\left|A_{n_{i_{0}}}^{f}(x)-\int f d \mu_{x}\right|<\varepsilon / 2,
$$

and for each $j=1, \ldots, q$,

$$
\left|A_{n_{i_{j}}}^{f}\left(T^{n_{i_{0}}+n_{i_{1}}+\cdots+n_{i_{j-1}}} x\right)-\int f d \mu_{x}\right|<\varepsilon / 2,
$$

while the last term $\frac{m}{n} A_{m}^{f}\left(T^{n_{i_{1}}+n_{i_{2}}+\cdots+n_{i_{q}}} x\right)$ has absolute value smaller than $\varepsilon / 2$. By averaging, we conclude that the inequality

$$
\left|A_{n}^{f}(x)-\int f d \mu_{x}\right|<\varepsilon
$$

holds for any $n>M$ uniformly for all $x \in X$, which implies uniformity.

The equivalence between strict uniformity and continuous strict pointwise ergodicity is the (just proved) equivalence between continuous pointwise ergodicity and uniformity, applied to semisimple systems.

The following diagram shows all the implications between the conditions discussed in this paper: (I)-(VI), (U), (sU), (S), (P) and (B):

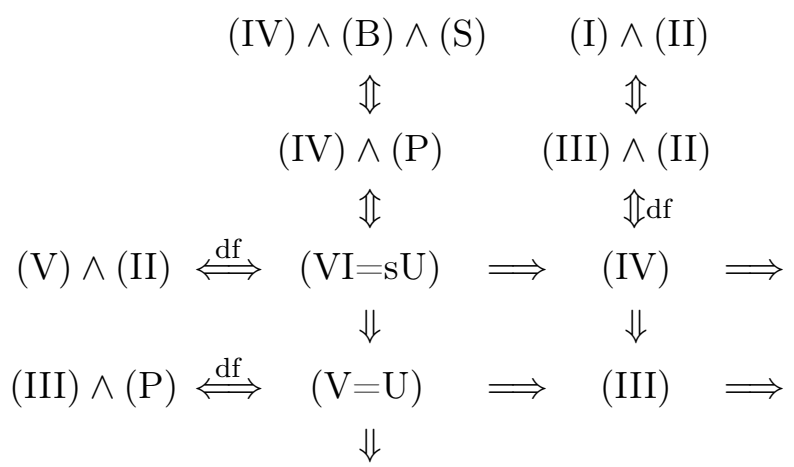

By analogy to the conditions equivalent to (VI), one might ask: is (V) equivalent to $(\mathrm{III}) \wedge(\mathrm{B}) \wedge(\mathrm{S})$ ? This question has a negative answer: as we will show by examples, neither $(\mathrm{III}) \wedge(\mathrm{B}) \wedge(\mathrm{S})$ implies $(\mathrm{V})$ nor $(\mathrm{V})$ implies $(\mathrm{S})$.

Nonetheless, the conjunction $(\mathrm{III}) \wedge(\mathrm{B}) \wedge(\mathrm{S})$ has a special meaning. Recall that in a uniquely ergodic system $(X, T)$, the unique minimal set $M$ pro- 
duces a strictly ergodic (hence strictly uniform) subsystem $(M, T)$ carrying the same invariant measure as $(X, T)$. Notice that if $(X, T)$ is partitionable then the union of all minimal sets (regardless of whether it is closed or not) supports the same invariant measures as $(X, T)$. Thus, a natural question arises: under what conditions does the union of minimal subsets of a partitionable system $(X, T)$ produce a strictly uniform subsystem? Below we give a complete answer.

TheOREM 4.10. Let $(X, T)$ be partitionable. Let $M$ denote the union of all minimal subsets of $X$. Then $M$ is closed and the system $(M, T)$ is strictly uniform if and only if $\mathcal{M}_{T}^{\mathrm{e}}(X)$ is compact and the set-valued function supp : $\mathcal{M}_{T}^{\mathrm{e}}(X) \rightarrow 2^{X}$ is continuous.

Proof. First notice that partitionability of $(X, T)$ implies that the family of all minimal subsets coincides with $\operatorname{supp}\left(\mathcal{M}_{T}^{\mathrm{e}}(X)\right)$, i.e. with the image of $\mathcal{M}_{T}^{\mathrm{e}}(X)$ via supp. Now assume that $\mathcal{M}_{T}^{\mathrm{e}}(X)$ is compact and supp : $\mathcal{M}_{T}^{\mathrm{e}}(X) \rightarrow 2^{X}$ is continuous. It follows that $\operatorname{supp}\left(\mathcal{M}_{T}^{\mathrm{e}}(X)\right)$ is compact, hence closed in $2^{X}$. This easily implies that the union $M$ of all minimal sets is closed in $X$. The system $(M, T)$ satisfies partitionability (III) and semisimplicity (II), so it is strictly pointwise ergodic (IV). It also satisfies (B) and $(\mathrm{S})$. Since $(\mathrm{IV}) \wedge(\mathrm{B}) \wedge(\mathrm{S}) \Leftrightarrow(\mathrm{sU}),(M, T)$ is strictly uniform.

Conversely, assume that $M$ is closed and that the system $(M, T)$ is strictly uniform. Then $(M, T)$ satisfies $(\mathrm{B})$ and $(\mathrm{S})$, i.e. $\mathcal{M}_{T}^{\mathrm{e}}(M)$ is compact and the function supp : $\mathcal{M}_{T}^{\mathrm{e}}(M) \rightarrow 2^{M} \subset 2^{X}$ is continuous. By partitionability, we have $\mathcal{M}_{T}^{\mathrm{e}}(M)=\mathcal{M}_{T}^{\mathrm{e}}(X)$, implying that the conditions $(\mathrm{B})$ and $(\mathrm{S})$ hold also for $(X, T)$.

We remark that without partitionability of $(X, T)$ (even with pointwise ergodicity assumed instead), Theorem 4.10 loses its valuable interpretation. The conjunction $(\mathrm{B}) \wedge(\mathrm{S})$ still implies that $M$ is compact and whenever $(M, T)$ is pointwise ergodic, it is strictly uniform. However, without partitionability, there is no guarantee that $\mathcal{M}_{T}(M)=\mathcal{M}_{T}(X)$ and $(M, T)$ cannot replace $(X, T)$ in the role of a topological model of some measure-preserving systems. This happens for instance in the example given by Katznelson and Weiss [KW] (see below for more details).

On the other hand, partitionability of $(X, T)$ does not follow from the assumptions that $(X, T)$ is pointwise ergodic, $M$ is compact, even if $(M, T)$ is strictly uniform and carries the same invariant measures as $(X, T)$. The simplest example here is $X$ being the two-point compactification of $\mathbb{Z}, X=$ $\mathbb{Z} \cup\{-\infty, \infty\}$, with $T x=x+1$. In this system $M=\{-\infty, \infty\}$ is closed and $(M, T)$ is trivially strictly uniform. Nonetheless, $(X, T)$ is pointwise ergodic without being partitionable; the set of points generic for $\delta_{\infty}$ equals $\mathbb{Z} \cup\{\infty\}$ and is not closed. 
5. Examples. One of the first examples showing phenomena associated to the notions discussed in the preceding section is given in [KW]. The system $(X, T)$ is pointwise ergodic but not partitionable. The set $\mathcal{M}_{T}^{\mathrm{e}}(X)$ is homeomorphic to the unit interval by a map $t \mapsto \mu_{t}$, in particular $\mathcal{M}_{T}^{\mathrm{e}}(X)$ is compact and uncountable. The topological supports of the ergodic measures form a nested family of sets $E_{t}$ intersecting at a fixpoint $x_{0}$ (which is a unique minimal subset). It can be verified that also the set-valued map $t \mapsto E_{t}$ is continuous (into $2^{X}$ ), implying that supp is continuous on $\mathcal{M}_{T}^{\mathrm{e}}(X)$. This example shows that $(\mathrm{I}) \wedge(\mathrm{B}) \wedge(\mathrm{S}) \nRightarrow(\mathrm{III})$.

We continue with six other examples. They are elementary, yet resolve possible questions about other implications. The space $X$ in the first five of them is the same and consists of a sequence of circles $C_{k}(k \geq 1)$ converging to a circle $C$ (in the last example we add to this space one more circle $C^{\prime}$ ). The circles $C_{k}$ and $C$ can be imagined as all having equal diameters and placed on parallel planes, or as concentric circles with radii of $C_{k}$ converging to the radius of $C$. On each circle $C_{k}$ we pick one point $c_{k}$ so that these points converge to a point $c \in C$. We will call the points $c_{k}$ and $c$ the origins of the circles $C_{k}$ and $C$, respectively (they will be used in Examples 5.2, 5.3 and 5.6.

EXAMPLE 5.1. In this example, the transformation $T$ restricted to each circle $C_{k}$ (henceforth denoted by $T_{k}$ ) is an irrational rotation. The angles of the rotations $T_{k}$ tend to zero as $k \rightarrow \infty$, so that $T$ restricted to $C$ is the identity map (see Figure 1).
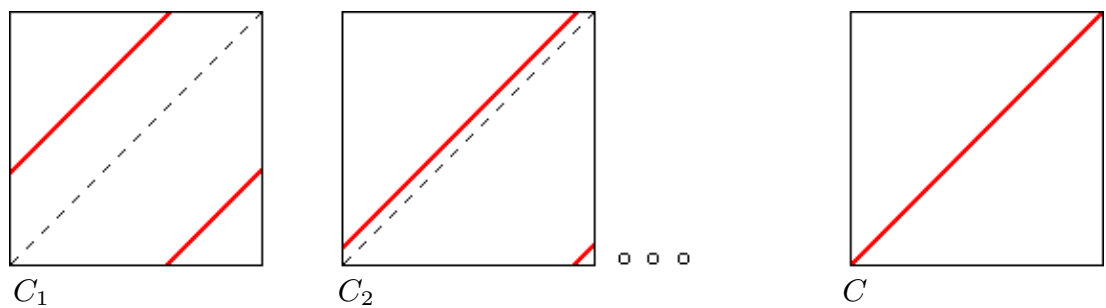

Fig. 1

Clearly, this system is strictly partitionable. The strictly ergodic subsystems are the circles $C_{k}$ and all single points on $C$. The set $\mathcal{M}_{T}^{\mathrm{e}}(X)$ consists of the normalized Lebesgue measures $\lambda_{k}$ on the circles $C_{k}$ and the Dirac measures $\delta_{x}$ at all points $x \in C$. This set of measures is not closed in $\mathcal{M}_{T}(X)$ (the measures $\lambda_{k}$ converge to the nonergodic Lebesgue measure on $C$ ). So, the system cannot be continuously pointwise ergodic and, by Theorem 4.9 , it is not uniform. It follows, that the partition $\mathcal{P}$ cannot be upper semicontinuous, and $\Phi: X \rightarrow \mathcal{M}_{T}(X)$ cannot be continuous (all these negative properties can be seen directly, but we want to show how our diagram works). On the 
other hand, the map supp is easily seen to be continuous, even on the closure of $\mathcal{M}_{T}^{\mathrm{e}}(X)$, which consists of two disjoint compact sets: one containing the Lebesgue measures on all the circles (including $C$ ) and the other consisting of all Dirac measures at the points $x \in C$. This example shows that without $(\mathrm{B}),(\mathrm{IV}) \wedge(\mathrm{S}) \nRightarrow(\mathrm{VI})$ (not even $(\mathrm{V}))$.

EXAMPLE 5.2. We slightly change the transformations $T_{k}$ in the previous example. For each $k$ we pick an arc $I_{k}$ containing the origin $c_{k}$. The diameters of the arcs decrease to zero, so that the arcs converge to the singleton $\{c\}$. On each $I_{k}$ we "slow down the motion", meaning that we locally bring the graph of $T_{k}$ closer to the diagonal (see Figure 2). Since $T$ is not changed outside the arcs $I_{k}$ (and on each $I_{k}$ the map is even closer to the identity than before), the maps $T_{k}$ converge, as before, to the identity map on $C$. We can easily arrange the modifications so that each $T_{k}$ is conjugate to some irrational rotation (much slower than before). We let $\mu_{k}$ denote the unique invariant (and ergodic) measure supported by $C_{k}$. Each orbit in $C_{k}$ now spends much more time in $I_{k}$ than before, so $\mu_{k}\left(I_{k}\right)$ is larger than $\lambda_{k}\left(I_{k}\right)$. By slowing down strongly enough we can easily arrange that $\mu_{k}\left(I_{k}\right) \rightarrow 1$, implying that the measures $\mu_{k}$ converge to $\delta_{c}$.
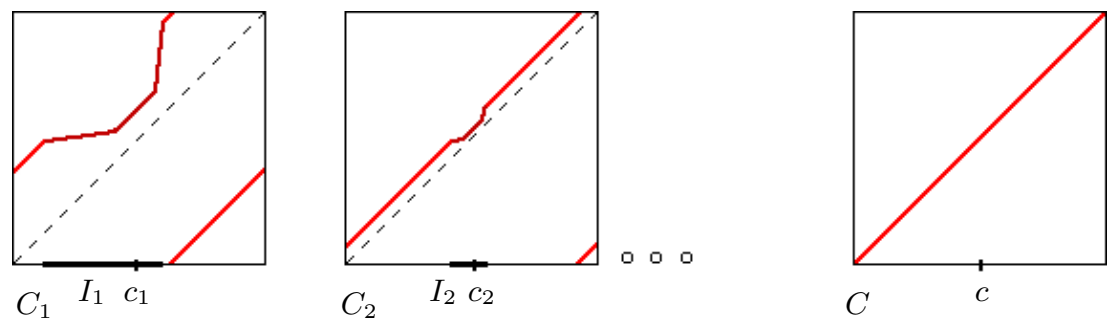

Fig. 2

The system is strictly partitionable with the same partition $\mathcal{P}$ as in the previous example. Since this partition is not upper semicontinuous, the system is not continuously pointwise ergodic. However, this time, the set of ergodic measures $\left\{\mu_{k}: k \geq 1\right\} \cup\left\{\delta_{x}: x \in C\right\}$ is closed in $\mathcal{M}_{T}(X)$ (because $\left.\mu_{k} \rightarrow \delta_{c}\right)$, hence compact. Since $(\mathrm{IV}) \wedge(\mathrm{B})$ holds and (VI) does not, it must be (S) that fails (this can also be seen directly: $\operatorname{supp}\left(\mu_{k}\right)=C_{k} \rightarrow C \neq \operatorname{supp}\left(\delta_{c}\right)$ ). This example shows that without $(\mathrm{S}),(\mathrm{IV}) \wedge(\mathrm{B}) \nRightarrow(\mathrm{VI})$ (not even $(\mathrm{V})$ ).

EXAMPLE 5.3. This is the declared example showing that $(\mathrm{III}) \wedge(\mathrm{S}) \wedge(\mathrm{B})$ $\nRightarrow(\mathrm{V})$. Let the map $T_{k}$ on the circle $C_{k}$ fix the origin $c_{k}$ and move all other points, say clockwise, but with the speed of movement decaying as $k$ increases, so that on $C$ we have the identity map (see Figure 3).

Clearly, the system is partitionable, with the same partition $\mathcal{P}$ as in Examples 5.1 and 5.2. Since $\mathcal{P}$ is not upper semicontinuous, the system 

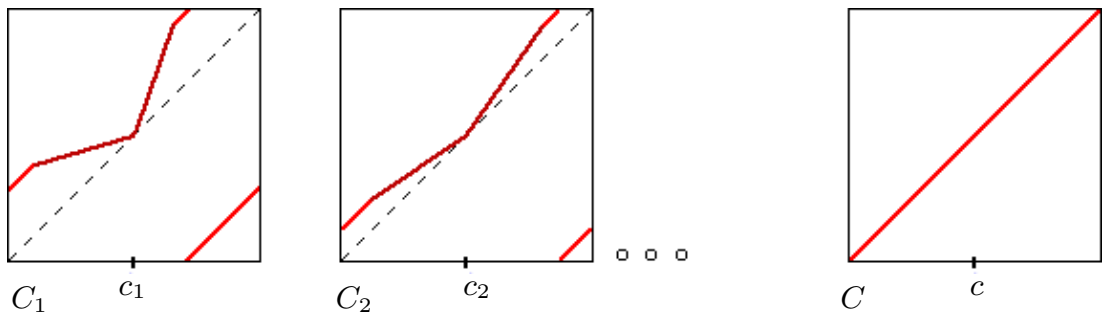

Fig. 3

is not continuously pointwise ergodic. The set of ergodic measures equals $\left\{\delta_{c_{k}}: k \geq 1\right\} \cup\left\{\delta_{x}: x \in C\right\}$ and is compact, and the set-valued function supp : $\mathcal{M}_{T}^{\mathrm{e}}(X) \rightarrow 2^{X}$ is obviously continuous.

EXAMPLE 5.4. This is the other declared example showing that $(\mathrm{V}) \nRightarrow(\mathrm{S})$ (and some other failing implications). The maps $T_{k}$ on the circles $C_{k}$ are modifications of one fixed irrational rotation. We can "slow down the motion" on each circle $C_{k}$ so that the resulting maps $T_{k}$ are conjugate to irrational rotations, and converge to a continuous map on $C$ which fixes just one point $c$ and moves all other points, say clockwise (see Figure 4). Then $\delta_{c}$ is the unique invariant (and ergodic) measure supported by $C$. The unique invariant (and ergodic) measures $\mu_{k}$ supported by $C_{k}$ must accumulate at invariant measures supported by $C$, so they have no choice but to converge to $\delta_{c}$.
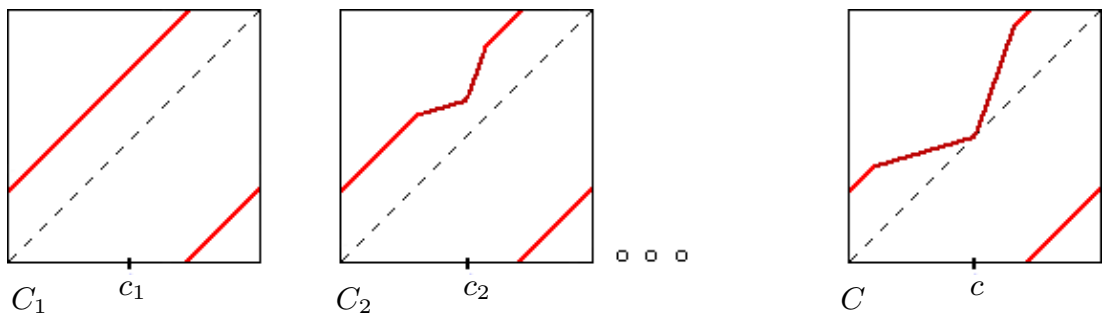

Fig. 4

The system is partitionable but not semisimple. The partition $\mathcal{P}$ into closed uniquely ergodic sets equals $\left\{C_{k}: k \geq 1\right\} \cup\{C\}$ and is continuous, so $(X, T)$ is continuously pointwise ergodic. In particular, $\mathcal{M}_{T}^{\mathrm{e}}(X)$ is compact (this can also be seen more directly, by noting that $\mathcal{M}_{T}^{\mathrm{e}}(X)=\left\{\mu_{k}: k \geq 1\right\}$ $\left.\cup\left\{\delta_{c}\right\}\right)$. However, since the supports of $\mu_{k}$ are whole circles, while that of the limit measure $\delta_{c}$ is a singleton, the function supp : $\mathcal{M}_{T}^{\mathrm{e}}(X) \rightarrow 2^{X}$ is not continuous. This example shows that $(\mathrm{V}) \nRightarrow(\mathrm{II})$ and $(\mathrm{V}) \nRightarrow(\mathrm{S})$. This example also shows that although (VI) implies continuity of $\mathcal{P},(\mathrm{V})$ combined with this continuity still does not imply (VI). 
EXAMPLE 5.5. Now we change the maps $T_{k}$ so that they all are the same as that on $C$ (see Figure 5).
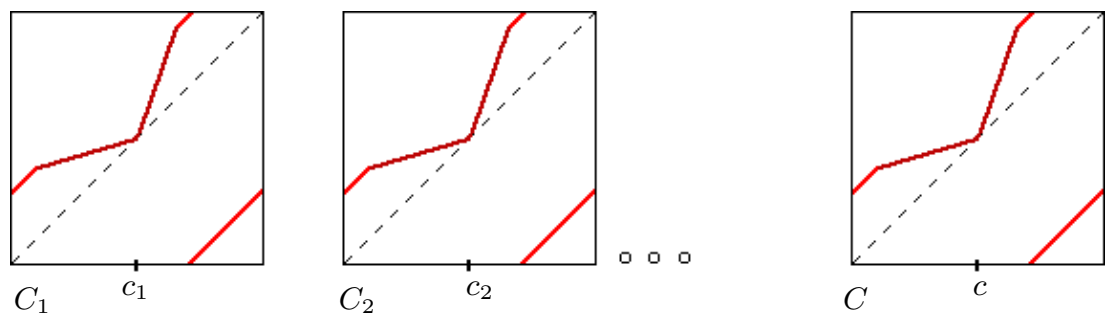

Fig. 5

As in the previous example, the system is partitionable but not semisimple, with $\mathcal{P}$ continuous, hence $(X, T)$ is continuously pointwise ergodic. This time the map supp: $\mathcal{M}_{T}^{\mathrm{e}}(X) \rightarrow 2^{X}$ is continuous. The example shows that $(\mathrm{V})$ combined with $(\mathrm{S})$ (and additionally with the continuity of $\mathcal{P}$ ) still does not suffice for (VI).

EXAMPLE 5.6. In the above example, we can add to the space one more circle $C^{\prime}$ that is tangent to $C$ at $c$. The dynamics on $C^{\prime}$ is symmetric to that on $C$, so that all points on $C^{\prime}$ are generic for $\delta_{c}$ and $C \cup C^{\prime}$ is an atom of $\mathcal{P}$ (see Figure 6).

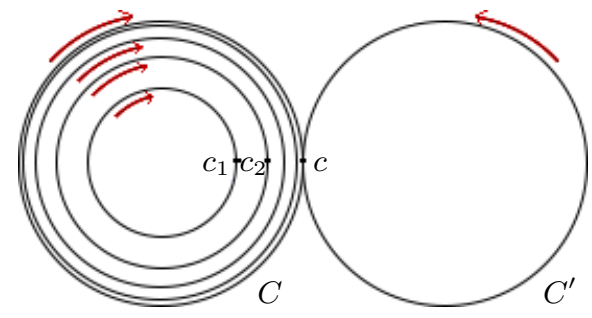

Fig. 6

The modified system has the same properties as in the preceding example, except that the partition $\mathcal{P}$ is now upper semicontinuous but not continuous. This example shows that (V) (in contrast to (VI)) does not imply continuity of $\mathcal{P}$, even when combined with $(\mathrm{S})$.

Acknowledgements. The authors thank Mariusz Lemańczyk for raising a question that triggered this research.

Tomasz Downarowicz is supported by National Science Center, Poland (Grant No. 2018/30/M/ST1/00061) and by the Wrocław University of Science and Technology (Subsidy for 2020, budget no. 8201003902 MPK: 9130730000). 


\section{References}

[H] G. Hansel, Strict uniformity in ergodic theory, Math. Z. 135 (1974), 221-248.

[HY] J. G. Hocking and G. S. Young, Topology, Addison-Wesley, London, 1961.

[J] R. I. Jewett, The prevalence of uniquely ergodic systems, J. Math. Mech. 19 (1970), 717-729.

[KW] Y. Katznelson and B. Weiss, When all points are recurrent/generic, in: A. Katok (ed.), Ergodic Theory and Dynamical Systems I, Progr. Math. 10, Birkhäuser, Boston, MA, 1981, 195-210.

[K] W. Krieger, On unique ergodicity, in: Proceedings of the Sixth Berkeley Symposium on Mathematical Statistics and Probability, Vol. II, Univ. of California Press, 1972, $327-345$.

[O] J. C. Oxtoby, Ergodic sets, Bull. Amer. Math. Soc. 58 (1952), 116-136.

Tomasz Downarowicz

Faculty of Pure and Applied Mathematics

Wrocław University of Technology

Wrocław, Poland

ORCID: 0000-0001-5732-7264

E-mail: downar@pwr.edu.pl
Benjamin Weiss

Einstein Institute of Mathematics The Hebrew University of Jerusalem Jerusalem, Israel ORCID: 0000-0002-2123-678X E-mail: weiss@math.huji.ac.il 